\title{
An overview on osteogenic differentiation process: Minimum essential information for bone tissue engineering
}

\section{Aida Șelaru*, luliana Samoilă*, Sorina Dinescu ${ }^{\bowtie}$, Marieta Costache}

Department of Biochemistry and Molecular Biology, Faculty of Biology, University of Bucharest, Spl. Independentei 91-95, 050095, Bucharest, Romania. *Both authors contributed equally to this manuscript.

${ }^{\bowtie}$ Correspondence to: Sorina Dinescu, Phone: 0213181575 Fax: 0213181575, int.102, E-mail: sorina.dinescu@ bio.unibuc.ro

Received: 4 December 2017 / Revised: 28 March 2018 / Accepted: 30 March 2018 / Available online: 15 April 2018

\begin{abstract}
Bone tissue engineering is a new and modern strategy that serves for repairing major bone defects. Three essential elements are involved in bone tissue engineering: a scaffold, a cell source that can convert into bone cells and growth factors. In order to develop new and original biomaterials with high compatibility it is necessary to fully understand osteoblastogenesis. Osteoblasts and osteoclasts are the two types of cells involved in the formation of bone tissue. Osteoblasts are cells responsible for bone growth and for synthesizing bone matrix, whereas osteoclasts function in bone resorbtion. There are some osteogenic markers that osteoblasts are able to produce during bone formation, that have an important role in the processes of migration, proliferation and differentiation. Also, the differentiation process is governed by three major signaling pathways: Wnt, Hedgehog and Notch, which have the role to regulate osteogenesis by controlling proliferation, differentiation and apoptosis. This review aims to synthesize the most important information known so far regarding the main stages and markers of osteogenesis, as well as the main biomaterials, major cell types and specific osteogenic inducers involved in bone tissue engineering. Moreover, this review can serve as a guideline for further applications concerning this subject.
\end{abstract}

Keywords: osteogenesis, osteogenic signaling pathways, bone tissue engineering, regeneration, transcription factors

\section{Introduction}

Bone tissue engineering (BTE) has emerged as a necessity for tissue production and aims to reconstruct the bone in the event of diseases that may interfere with bone function or major bone injuries. Biomaterials, cells, and induction factors are required to carry out a reconstruction process in tissue engineering (TE). It is preferred that the biomaterials exhibit a corresponding bone-like composition and allow the cells to proliferate and differentiate appropriately to osteoblasts.

For this strategy to work, it is not possible to use any type of cell, so only cells that are or may be programmed to differentiate into bone cells are used, such as cell lines of preosteoblasts which are committed to the osteogenic fate or stem cells (induced pluripotent stem cells, mesenchymal stem cells (MSCs), adipose-derived stem cells). A common problem that requires increased attention is the quality of the cells used. As a result, it has been proven that the stem cells from young donors present a considerably higher differentiation efficiency compared to those from older donors (Harrt et al., 2012). Last but not least, the presence of inducing and growth factors is necessary, because they are responsible for triggering signaling pathways specific for the osteogenic differentiation.

Even though a lot of abundant medical abilities were discovered, researchers still have much to study in the field of BTE, studies relying on the combination of bioengineering, clinical medicine, biomedical science and stem cell biology (Badylak and Nerem, 2010).

\section{Bone tissue engineering (BTE)}

BTE combines the knowledge of molecular biology and cell biology, as well as the various field of materials useful in regenerating tissues (Williams, 2004). Also, it combines the knowledge of the principles of life sciences with those of engineering for a good understanding of the relationships between structure and function in tissues. This work is vital in the development of tissues to replace the old ones (Hubbell, 1995).

The basis of TE is the ability of cells to initiate and maintain the regeneration process by the activation of particular genes under the influence of inducers (Williams, 2004). 
The goal of regenerative medicine is maintaining, refining and improving a defective tissue (Pandolfi et al., 2016). The bone is a dynamic tissue which is capable of regenerating after small injuries. But when it comes to more severe wounds which result after traumas, malformation and tumors the bone remodeling capacity is limited. Therefore, the intervention of BTE can be the answer for healing serious injuries. BTE principle (Figure 1) is based on combining three essential elements: (1.) a scaffold which resembles the structure of the bone matrix, (2.) a cell source with potential to convert to bone cells and (3.) inductive growth factors (Rose et al., 2002).

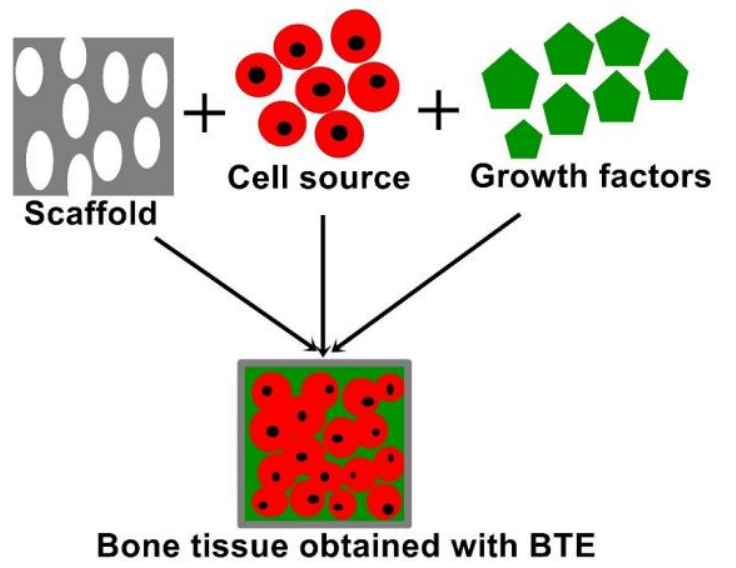

Fig. 1. Principle of BTE

Biomaterials or scaffolds are temporary materials that are designed to serve as architectural support for cells in order to grow or regenerate the bone. Bone tissue consists of several cell types: osteoprogenitor cells, osteoclasts, osteocytes and osteoblasts, each type having a specific role in bone tissue homeostasis. Both osteoblasts and mature osteocytes are responsible for synthetizing the mineralized bone matrix. This matrix serves as a reservoir of water, nutrients, cytokines and growth factors, thus allowing cells to adhere to the substrate (Kneser et al., 2002). In practice, a wide range of materials have been used as matrices in BTE. Over the years, these have been optimized taking into account the following properties: porosity, pore size and surface properties (Salgado, 2004).

The most important quality a scaffold is required to present for successful BTE is biocompatibility. The cells need to adhere, function normally, migrate on the surface of materials and inside the scaffolds, and be able to proliferate. In addition, implanted biomaterials should allow the body's own cells to populate the implant in order to start replacing the material with de novo synthesized tissue. Thus, another important feature results, namely biodegradability (Babensee et al., 1998). Products resulting from biodegradation should not be toxic and should be capable of leaving the body without interacting or affecting other organs (O'Brien, 2011).

Vascularization plays an important role in the transport of oxygen and nutrients to cells and in the export of waste from the tissue construct. This process is especially important in TE with three-dimensional (3D) structure organs, such as the heart, kidney and liver, but its importance has also been demonstrated in muscle, nervous system and bone tissue. In the case of BTE, following steps have been taken into consideration to help the vascularization within the construct: directing cellular behavior by growth factors, using a co-culture system, applying mechanical stimulation, using biomaterials with appropriate properties and incorporating microfabrication techniques. Interaction between cells plays an important role in targeting them to proliferation and differentiation processes. Moreover, osteoclasts are dependent on the attachment to the bone matrix in order to undergo differentiation (Feng, 2009). Therefore, the interaction between cells and extracellular bone matrix is also crucial for proliferation and differentiation. In vivo, the interaction between cells takes place via signaling cascades. During the bone repair process, growth factors have an impact on cell migration, proliferation and differentiation. Bone morphogenetic protein 2 (BMP-2), together with transforming growth factor $\beta$ (TGF- $\beta$ ), fibroblast growth factor (FGF), platelet derived factor (PDGF), insulin growth factor (IGF) endothelial growth (VEGF) are all involved in the bone repair process (Devescovi et al., 2008; Nguyen et al., 2012). BMP-2, PDGF, FGF and VEGF have the ability to stimulate the migration process of osteoprogenitor cells, whereas TGF- $\beta$, IGF and BMP modulate proliferation and differentiation. In addition, VEGF and FGF have the role of initiating vascular growth during bone formation (Nguyen et al., 2012).

First attempts in bone reconstruction were developed using bone grafts. Studies have divided these grafts into three categories: autogenous, allogenic and heterologous (Nga et al., 2015). Autografts are considered to be the perfect solution when it comes to bone regeneration because of their favorable osteoinductive and osteoconductive properties and because they are histocompatible and non-immunogenic (Amini et al., 2012; Neen et al., 2006). The major issue in this case is the fact that an autologous bone transplant is very expensive and the donor can suffer from morbidity at the injured site (Amini et al., 2012; Banwart et al., 1995; Goulet et al., 1997). An alternative to this procedure is the allogeneic bone graft, but this kind of technique meets the usual transplant risks: the rejection of the implant and the transfer of pathogen agents from the donor to the patient (Betz et al., 2002). Because of all these disadvantages and restrictions, this procedure is no longer considered a BTE strategy and, therefore, it led to finding new solutions for bone regeneration. The production of synthetic and natural biomaterials, combined with stem cells and inducers under controlled conditions, is known to be the BTE strategy (Burny et al., 1995). 


\section{Osteogenic differentiation}

The formation of bone tissue involves three processes: osteogenesis, modeling and remodeling. Osteogenesis is defined as the process in which undifferentiated cells or bone precursors become osteoblasts and later on osteocytes and osteoclasts. Bone modeling is responsible for maintaining the bone structure by removing existent damages and changing bone shape and size (Seeman, 2009). The remodeling process includes three distinct phases: the digestion of old bone by osteoclasts, the appearance of cells on the bone surface and the renewal of the bone (Hadjidakis and Androulakis, 2006). Osteoblasts together with osteoclasts are responsible for mediating these processes (Rutkovskiy et al., 2016). The differentiation process of stem cells into mature osteocytes includes more steps: (1.) the engagement of the cells on the osteogenic differentiation pathway, (2.) cell proliferation which leads to the formation of osteoprogenitor cells, (3.) the development of osteoprogenitor cells to preosteoblasts and (4.) the mineralization of the matrix which is produced by mature osteoblasts and osteocytes (Jayakumar et al., 2010).

The MSCs present a multipotent character which underlies their capacity to give rise to myoblasts, osteoblasts, chondrocytes and adipocytes (Rutkovskiy et al., 2016). Each lineage is controlled by so called "master transcriptional regulators" (Rutkovskiy et al., 2016), that are essential for each differentiation process. Myoblast Determination Protein is for the myoblast lineage, Sex determining region of the Y-box 9 is for the chondroblast lineage, Peroxisome proliferator-activated receptor gamma for the adipocyte lineage and Runt-related transcription factor 2 (RUNX2) for the osteoblast lineage (Rutkovskiy et al., 2016).

As soon as RUNX2 is activated, the cells begin the differentiation process undergoing more stages, each stage being characterized by particular molecular markers (Rutkovsky et al., 2016). First, cells engage in the osteogenic lineage and are defined as osteoprogenitor cells (Jayakumar et al., 2010). Next, they continue their proliferation towards committed pre-osteoblasts which start expressing alkaline phosphatase (ALP), which is known to be an early marker in this process (Luu et al.,2007). A phenotypic change takes place when preosteoblasts become mature osteoblasts: a larger nucleus and Golgi, the endoplasmic reticulum expands to support the secretion of collagen type I (COLI) (Luu et al.,2007). Then, cells begin to synthesize the extracellular matrix which is maturated with COLI and fibronectin. The matrix mineralization takes place in the last stage when the osteopontin (OPN) and osteocalcin (OCN) (late differentiation markers), responsible for promoting the deposition of mineral matrix, are expressed. At the end of this biological process osteoblasts become osteocytes, which are found in a lacunar portion of the bone and, due to their extensions, they form a network providing mechanical support (Luu et al.,2007).

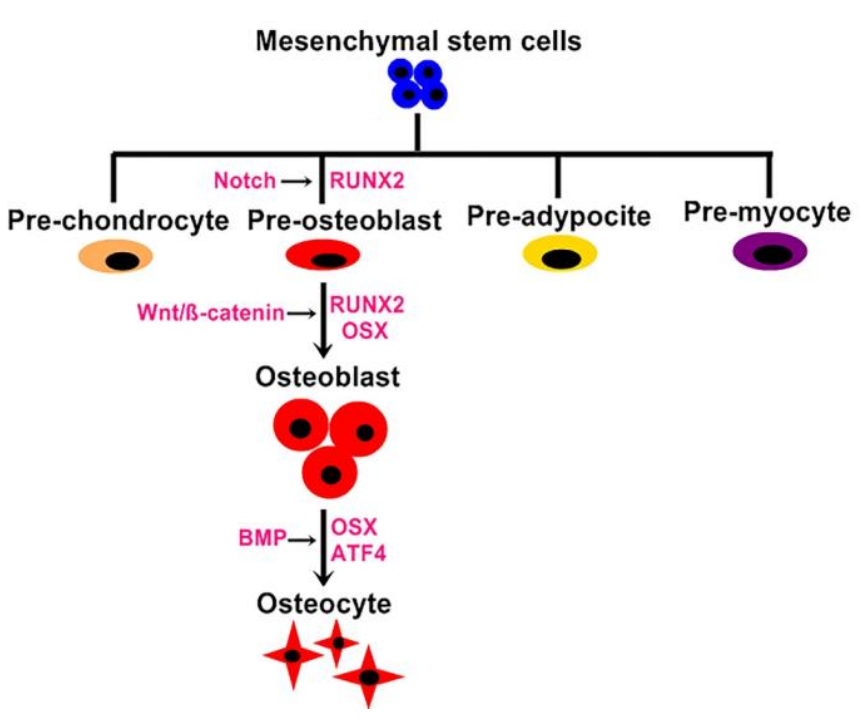

Fig. 2. Notch signaling pathway is present at early stages of osteoblast differentiation by acting on RUNX2 gene expression. Wnt/ $\beta$-catenin signaling pathway promotes preosteoblast differentiation, interfering with RUNX2 and OSX, which leads to differentiation and mineralization processes. BMP signaling is required so that osteocyte differentiation can be possible in the final stages of bone formation. This signaling pathway uses transcription factors, such as OSX and ATF4, for osteocyte differentiation and maturation.

During embryonic skeletal formation and growth, osteoblasts reach the highest level of activity. They remain present throughout life, but are not as active as they are during embryogenesis. Only in case of an injury in an adult organism osteoblasts are activated for the regeneration process (Rutkovskiy et al., 2016). Osteoblasts have a slow response: it takes four months for a mature osteoblast to synthesize bone matrix (Rutkovskiy et al., 2016). Osteoblasts are post-mitotic cells (Long, 2012), but their differentiation is not yet finalized. The ones that develop the ability to produce mineralized bone matrix will differentiate to osteocytes. The proteins contained in the extracellular matrix are expressed and secreted by osteoblasts forming the osteoid, which is also known as the non-mineralized bone matrix. Osteoid proteins are cross-linked during matrix maturation, thus forming a stable structure. Cross-linked COL1 fibers, the major component of the osteoid, represent the support for deposing inorganic minerals and, as result, the mineralized bone matrix is formed. At the end of this stage, osteoblasts incorporated in their own secreted matrix become osteocytes, which diminish the rate of bone matrix production (Jayakumar et al., 2010). Osteocytes are the most abundant cells of the bone matrix and have a stellar shape and are interconnected, forming a network, being responsible for the turnover of the bone material (Rutkovskiy et al., 2016). When the population of osteocytes decreases, new osteoblasts are differentiated from MSCs even though their resource is limited (Rutkovskiy et al., 2016). 
Osteoclasts are big, multinucleated cells which are attached to the bone matrix via a brush border which is responsible for solubilizing the mineral content increasing the number of protons. This process is really well stabilized through the paracrine co-regulation between osteoblasts and osteoclasts in a process called bone remodeling (Polo-Corrales et al., 2014). Bone matrix proteins are composed of collagen and noncollagen proteins, which include glycoproteins, proteoglycans and growth factors. Collagen constitutes $90 \%$ of the total weight of bone matrix proteins and is mostly represented by COLI $(97 \%)$, but small amounts of collagen type III, V, IX, XII are also to be found. The non-collagen proteins constitute $10 \%$ of the bone mass (Gentili and Cancedda, 2009). The glycoproteins include ALP, OCN, OPN and bone sialo protein, which modulate the mineralization process, and osteonectin which modulates the diameter of the collagen fibers. The bone matrix contains a large number of proteins that have an integrin binding domain (RGD) such as, thrombospondin, fibronectin, vitronectin, fibrillin 1 and 2, OPN, COLI and bone sialo protein (Clarke, 2008). Proteoglycans are regulators of growth factors, presenting the capacity to store them in the bone matrix. This may lead to an increased concentration of growth factors in the bone and can be further used in the differentiation process (Lamoureux et al., 2007).

\section{Genes and proteins in osteoblastogenesis}

The lineage fidelity of MSCs is managed by the expression of so-called master transcriptional regulators. Hence, Runt-related transcription factor 2 and osterix have an important role in osteoblast differentiation. After the activation of osteogenic cascade, Distal-less homeobox 5 and Core Binding Factor a-1 genes will also be activated. In the end, the cells will express Activating transcription factor 4 and osteopontin, which is responsible for mineralization of the extracellular matrix (Jensen et al., 2010).

\section{a. Distal-less homeobox 5}

Distal-less homeobox 5 (DLX5) has a very important role in the process of differentiation - it is responsible of producing skeletal elements and of osteogenesis in the development of hard tissue (Heo et al., 2017). In the absence of DLX5, MSCs are not able to differentiate into osteoblasts. Furthermore, it was observed that MSCs maintained in an osteogenic environment, but lacking DLX5, did not express BMP2. Yet, with or without the expression of BMP2 and DLX5, RUNX2 was regularly expressed. Therefore, RUNX2 initiation is not correlated with the induction of DLX5 or BMP2 in MSCs (Heo et al., 2017).

\section{b. Core Binding Factor a-1}

Core Binding Factor a-1 (CBFA1) is one of the many transcriptional activators of osteoblast differentiation. It has the power to control the main structural proteins of the bone matrix (Levi et al., 2002). In a study, mice who were deficient in CBFA1 lacked endochondral and intramembranous ossification due to the blockage osteoblast maturity (Komori and Kishimoto, 1998). It has been demonstrated that CBFA1 plays an important role in differentiation processes because it is present in both differentiated cells and their progenitors. In intramembranous ossification, where chondrocytic stage does not exist, condensing cells display high levels of CBFA1 and differentiate straight towards adult osteoblasts (Ducy, 2000). Although CBFA1 can influence bone formation after fetal period, it has a smaller impact after birth. The repair response that appears during osteogenesis or wound healing might be more dominant than the process during fetal bone formation (Isefuku et al.2004).

\section{c. Runt-related transcription factor 2}

Over time, RUNX2 was often found in murine thymus and $\mathrm{T}$ cells, while other tissues such as heart, liver, brain and kidney did not express RUNX2. Following these findings, it has been elaborated a hypothesis that RUNX2 is a T cell - specific transcriptional regulator. After more studies, it has finally been confirmed that, in vivo, RUNX2 has a central role in osteoblast differentiation (Figure 2) and bone formation (Bruderer et al., 2014). Also, it helps MSCs to differentiate into osteoblasts and it inhibits their transformation in other cell types, such as adipocytes or chondrocytes (Komori, 2006). RUNX2 was first discovered in preosteoblasts and its expression was stimulated in young osteoblasts and inhibited in mature osteoblasts (Komori, 2010). It is known to function as a regulator in osteoblast differentiation and has an important role in skeletal morphogenesis, chondrogenesis and tooth development. Also, it has a powerful epigenetic domination over the ribosomal RNA and can regulate G0/G1 evolution in osteoblast cells (Vimalraj et al., 2015). Furthermore, RUNX2 can control bone genes and chromatin remodeling factors by various interactions. It has been demonstrated that RUNX2 has a positive effect on osteoclast differentiation, chondrocyte differentiation, periosteal bone formation and vascular invasion (Vimalraj et al., 2015).

\section{d. Osterix}

Bone formation and in particular tooth formation are common processes in which the expression of osterix (OSX) is required. OSX is responsible for odontoblasts presence, by playing important roles in maturation and differentiation of preodontoblasts. Likewise, OSX is involved in differentiation of preosteoblast to osteoblasts (Sinha and Zhou, 2013). Studies concerning bone wounds have proved that OSX is strongly expressed during bone cells regeneration. OSX has an important role in expression of osteoblast genes (Figure 2) in the adult and in the evolution of adult bones. Also, OSX plays an essential part in deterioration of cartilage matrix by being 
involved in calcification of cartilaginous matrix (Sinha and Zhou, 2013).

\section{e. Osteopontin}

Osteopontin (OPN) is synthesized by preosteoblasts, osteoblasts and osteocytes. OPN is a significant component in bone formation, judging by its presence in early stages of embryogenesis (Butler, 1989). OPN has a significant role in accelerating recovery of the organism after injury, which is usually followed by a boost in its expression. OPN activates cellular signaling pathways through different receptors found on almost all types of cells. Furthermore, OPN can stimulate and participate in cell migration and it can inhibit apoptosis (Sodek, 2000).

\section{f. Activating transcription factor 4}

Activating transcription factor 4 (ATF4) is fundamental in late phases of osteoblast differentiation (Figure 2.) and it is located downstream of RUNX2. These two are required to enable OCN expression, because they make a complex at the promoter of OCN. Small amounts of OCN can be found in mice that do not express ATF4, whereas OCN increases proportionally with ATF4 (Rutkovskiy, 2016).

\section{Growth and transcription factors in osteogenic differentiation}

During osteogenesis process there are some molecules that can influence the cellular differentiation process, such as transcriptional factors and growth factors. The latter have a limited effect and are present in low concentrations. BTE has the purpose to integrate these transcriptional factors and growth factors working as inducers in scaffolds structure, for later usage in case of injuries (Andrades et al., 2013). Even though growth factors and signaling pathways work together, the mechanism of interaction is not completely yet understood (Hughes et al., 2006).

\section{a. Transforming growth factor $\beta$}

Superfamily TGF has more than 40 members and it is divided into four subfamilies. Subfamily TGF- $\beta$ can be only seen in mammals and it has three isoforms: TGF$\beta 1$, TGF- $\beta 2$ and TGF- $\beta 3$. The three isoforms have two components: mature TGF- $\beta$ and latency-associated protein. TGF- $\beta 1$ is a very common cytokine in the bone matrix. In case of tissue damage, TGF- $\beta 1$ can be stimulated by cleavage of latency-associated protein by osteoclasts (Crane and Cao, 2014). When it is active, TGF- $\beta$ can have numerous effects, including proliferation, migration, differentiation and apoptosis. These effects can be different depending on the cell type, because even though TGF- $\beta$ signaling can not induce osteoblast differentiation, it can lead to MSCs migration (Crane and Cao, 2014).

\section{b. Bone morphogenetic proteins}

Bone morphogenetic proteins (BMPs) are cytokines, usually necessary in postnatal ossification. They are members of the TGF- $\beta$ superfamily and they can send a signal by binding the ligand to the receptor of a cell surface (Rutkovskiy, 2016). BMPs can be split in 4 subfamilies: BMPs 2 and 4, BMPs 5, 6, 7, 8a and 8b, BMPs 9 and 10, and BMPs 12, 13 and 14. Each subfamily has a different sequence and function. For example, BMP2, BMP4 and BMP7 can be present in dental epithelium, whereas BMP8 and BMP9 can be involved in cartilage formation and BMP10 plays a key role in trabecular evolution in the embryo heart and oocyte evolution (Carreira et al., 2014). BMPs can contribute to the RUNX2 location upstream of transcription site, but also they can regulate transcription of its target genes by being paired with RUNX2 . Expression of OSX is not possible without the presence of BMP2 (Rutkovskiy, 2016). There are a few types of cells that can produce BMPs, cells such osteoblasts, chondrocytes, osteoprogenitor cells or endothelial cells. Also, BMPs can be found for a short period in extracellular matrix. It can be stored only briefly because they have to be released in case of bone reconstruction (Carreira et al., 2014).

\section{c. Parathyroid hormone}

Parathyroid hormone (PTH) is an upstream regulator of RUNX2. As the amount of hormone is getting higher, bone resorption starts. When the amount of hormone is raising in a discontinuous way, osteoblast differentiation occurs. This might happen because discontinuous raising can lead to release of TGF- $\beta$ from resorbing bone (Rutkovskiy, 2016). Cytokines and growth factors are released from the bone matrix at the same time as PTH is an intermediate for osteoclastic bone resorption (Crane and Cao, 2014). Preserving the skeleton, PTH helps controlling bone remodeling by coordinating signaling of local factors, such as TGF- $\beta$ or BMP (Crane and Cao, 2014).

\section{d. Fibroblast growth factor}

Fibroblast growth factors (FGF) are a structural polypeptide family. Receptors like FGFR1, FGFR2, FGFR3 and FGFR4 mediate FGF in their role in cellular proliferation, angiogenesis, differentiation and migration. FGF receptors have two domains: an extracellular immunoglobulin-like domain and a tyrosine kinase cytoplasmic activity domain ( $\mathrm{Ng}$ et al., 2008). In adult tissues, the most frequently found proteins are FGF1 and FGF2 (Canalis et al. 1988). In case of fracture healing, FGF and FGFR expression are monitored and there is proof that FGF/FGFR signaling has a very important role in the course of fracture repair. FGF2 is usually used to improve bone reconstruction in animals. Also, FGF2 can influence bone resorption by having a side effect on osteoclast precursor proliferation (Ornitz and Marie, 
2015). FGF also encourages the expression of CBFA1/RUNX2, ALP and COLI in osteoprogenitors (Bai et al., 2013). FGF treatment is influenced by the amount of dose needed, the moment and the period of application (Ornitz and Marie, 2015).

\section{e. Insulin-like growth factor}

Insulin-like growth factor (IGF) has a meaningful role in cellular proliferation and it enhances the adult osteoblast functions (Celil et al., 2005). IGF-1 is one of the factors that is very often found in the bone matrix and it is the most important factor that mediates bone remodeling. It has been demonstrated that, unlike the healthy population, people tend to have a smaller bone mass in diseases caused by low IGF-1 concentrations (Crane and Cao, 2013). IGF-1 helps bone remodeling by having the ability to intensify expression of genes that are important in stimulating cell migration. Over time, numerous studies had shown that some factors such as PTH, estrogen, GH and BMP2 are able to stimulate IGF-1 transcription, whereas other factors, such as PDGF, FGF and glucocorticoids can help inhibit IGF-1 transcription in osteoblasts. Also, these factors can be correlated with a higher or smaller amount of bone mass (Crane and Cao, 2013).

\section{f. Other transcription factors}

The transcription factors stated above develop an important part of the systems involved in the differentiation of osteoblasts. Despite the fact that regulatory systems still need to be further characterized, there are many other transcription factors involved in the differentiation process (Kirkham and Cartmell, 2007). Homeobox protein MSX-2 and Homeobox protein MSX1, homeobox transcription factor DLX6, Twist related protein, activated protein 1, knotted-like homeobox proteins and specificity protein 3. Homeobox protein MSX-2 and Homeobox protein MSX-1are proteins involved in the formation of the skull in murine, however there is controversy over the effect that they have on osteoblast differentiation. DLX6 is a protein expressed in osteoblasts throughout the body skeleton. Twist proteins are helix-loop-helix proteins and inhibit osteoblast differentiation. Activated protein 1 plays an important role in bone formation, but over-expressions of this protein are characteristic to osteoporosis. Knotted-like homeobox proteins and specificity protein 3 occur only in the terminal ossification phases (Komori, 2006).

\section{Signaling pathways involved in osteogenesis}

Beside all these molecules and growth factors which modulate the osteogenic differentiation, this process is regulated by a series of specific signaling pathways. All these are put in a complex combination in order for MSCs to become mature osteoblasts. The major signaling pathways which modulate the differentiation, proliferation and migration of cells during osteogenesis are Wingless-related integration site (Wnt), Hedgehog and Notch signaling pathways. Other than the role in the osteogenic differentiation, both Wnt and Hedgehog take part in adipogenesis, Wnt signaling pathway presenting an anti-adipogenic activity (James, 2013).

\section{a. Wnt signaling pathway}

In adult tissue, Wnt regulates osteogenesis by playing an important role in the renewal of $\mathrm{MSCs}$, in bone formation, specifically in osteoblast differentiation (Figure 2) and mineralization process and in cell adhesion and migration through indirect contact with cadherins (Arnsdorf et al., 2009). Abnormal activities of the signaling pathway have been associated with human bone diseases such as van Buchem's disease, sclerosteosis and osteoporosis (Monroe et al., 2012). Wnt is responsible for activating three different signaling cascades: Wnt/ $\beta$-catenin pathway, Wnt/Ca ${ }^{2+}$ pathway and Wnt/planar polarity pathway. The $\mathrm{Wnt} / \mathrm{Ca}^{2+}$ pathway is known as the non-canonical pathway and the Wnt/ $\beta$ catenin as the canonical Wnt pathway (Hayrapetyan et al., 2015). Concerning their role and therapeutic potential in bone disease, the canonical signaling pathway has been better characterized (Kim et al., 2013). Moreover, a series of studies have demonstrated the importance of the canonical Wnt pathway in promoting osteogenesis in vivo and in vitro (Chung et al., 2009). Quarto et al. (Quarto et al., 2010) evidenced that canonical Wnt signaling pathway can inhibit or promote osteogenesis, depending on the cell differentiation stage or on Wnt ligand concentration. During the osteogenic differentiation this signaling pathway interferes with RUNX2 and OSX (Hill et al., 2005).

\section{b. Hedgehog signaling pathway}

The Hedgehog family is composed of the Hedgehog protein and its homologous: Sonic hedgehog (Shh), Indian hedgehog (Ihh) and Desert hedgehog (Dhh). Indian hedgehog is produced by chondrocytes, acts directly on osteoblastic progenitors which have a perichondral localization (Long et al., 2004) and appears later in the enchondral bone formation of the limb. On the other hand, Sonic hedgehog appears in the early stages of bone development and is directly involved in the formation of the limb (Yang et al., 2015). Hedgehog activates in vitro the formation of osteoblasts (Long et al., 2004; Nakamura et al., 1997; Van der Horst et al., 2003). Indian hedgehog regulates endochondral ossification, by acting as a paracrine modulator in this process (Ehlen et al., 2006). The function of Indian hedgehog in bone length growth is mediated by peptideassociated parathyroid hormone (Vortkanp et al., 1999). Peptide-associated parathyroid hormone induces proliferation and reduces chondrocyte hypertrophy (Kronenberg, 2006). 


\section{c. Notch signaling pathway}

Notch signaling plays an important role in the development and homeostasis of adult tissue by regulating cell proliferation, differentiation and apoptosis. Notch receptors are transmembranar proteins that require contact with neighboring cells in order to induce signal transmission (Engin and Lee, 2010). Notch signaling is also involved in skeletal formation. This signaling pathway is present at early stages of osteoblast differentiation (Figure 2) by acting on RUNX2 gene expression (McLarren et al., 2000; Tezuka et al., 2002). Notch can regulate osteoclastogenesis, resulting in an over-expression of the receptor activator of nuclear factor- $\mathrm{\kappa B}$ ligand and osteoprotegerin. Communication between osteocytes and osteoclasts can be mediated through the Notch signaling pathway (Engin and Lee, 2010). Notch signaling is also involved in chondrogenic differentiation, although the role during chondro / osteogenesis has not been yet elucidated (Lattanzi and Bernardini, 2012). Moreover, this pathway is an essential sign for the debut of formation and maturation process of osteoclasts. It has been demonstrated that this signaling can prevent the formation of mature osteoclasts (Sethi and Kang, 2012; Hilton et al., 2008).

\section{Conclusions}

The osteogenic differentiation is a complex biological process which involves lots of molecules and signaling pathways and, because of its complexity, it has not been yet fully understood. What has been established until now is that osteoblasts have their origin in MSCs along with myoblasts, adipocytes and chondrocytes, which makes the osteogenic process difficult to activate. While engaging and promoting the osteogenic lineage, cells also have to compete against the other lineages in order to inhibit them. Moreover, the reserve of bone cells is limited and this can lead to serious disease such as osteopenia. In order to be able to bring solutions for serious bone injuries, traumas and disease, all these aspects have to be taken in consideration when trying to develop new matrices for BTE.

\section{Acknowledgments}

The Authors acknowledge their formation in the field of tissue engineering and cell differentiation by 65PCCDI project and PCCE 248/2010 national grant. No funding has been received for the preparation of the manuscript.

CThe Author(s) 2018

Open Access This article is distributed under the terms of the Creative Commons Attribution 4.0 International License (http://creativecommons.org/licenses/by/4.0/) which permits unrestricted use, distribution, and reproduction in any medium, provided you give appropriate credit to the original author(s) and the source, provide a link to the Creative Commons license, and indicate if changes were made.

\section{References}

Amini A. R., Laurencin C. T., Nukavarapu S. P. 2012. Bone tissue engineering: recent advances and challenges. Crit. Rev. Biomed. Eng. 40, 363-408.

Andrades J.A., Narváez-Ledesma L., Cerón-Torres L., Cruz-Amaya A.P., Daniel López-Guillén D., Laura Mesa-Almagro M.L., Moreno-Moreno J.A. 2013. Bone Engineering: A Matter of Cells, Growth Factors and Biomaterials. In Regenerative Medicine and Tissue Engineering, Prof. Andrades J.A. (Ed.) InTech, doi: $10.5772 / 56389$.

Arnsdorf E. J., Tummala P., Jacobs C. R. 2009. Noncanonical Wnt signaling and $\mathrm{N}$-cadherin related betacatenin signaling play a role in mechanically induced osteogenic cell fate. Plos One 4, e5388.

Babenseea J.E., Andersonb J.M., McIntirea L.V., Mikosa A.G. 1998. Host response to tissue engineered devices. Adv. Drug Deliv. Rev. 33, 111-139.

Badylak S.F., Nerem, R.M. 2010. Progress in tissue engineering and regenerative medicine. Proc. Natl. Acad. Sci. U.S.A. 107, 3285-3286.

Bai Y., Yin G., Huang Z., Liao X., Chen X., Yao Y., Pu $X$. 2013. Localized delivery of growth factors for angiogenesis and bone formation in tissue engineering. Int. Immunopharmacol. 16, 214-223.

Banwart J. C., Asher M. A., Hassanein R. S. 1995. Iliac crest bone graft harvest donor site morbidity. A statistical evaluation. Spine J. 20, 1055-60.

Betz R.R. 2002. Limitations of autograft and allograft: New synthetic solutions. Open Orthop. J. 25, 561-570.

Brien, F.J.O. 2011. Biomaterials \& scaffolds: Every day thousands of surgical procedures are performed to replace. Mater. Today 14, 88-95.

Bruderer M., Richards R. G., Alini M., Stoddart M. J. 2014. Role and regulation of RUNX2 in osteogenesis. Eur. Cell Mater. 28, 269-86.

Burny F., Donkerwolcke M., Muster D. 1995. Biomaterials education: a challenge for medicine and industry in the late 1990s. Mater. Sci. Eng. A, 199, 5359.

Butler, W. T. 1989. The nature and significance of osteopontin. Connect. Tissue Res. 23, 123-136.

Canalis E., Centrella M., McCarthy T. 1988. Effects of basic fibroblast growth factor on bone formation in vitro. J. Clin. Invest. 81, 1572-1577.

Carreira A. C., Lojudice F. H., Halcsik E., Navarro R. D., Sogayar M. C., Granjeiro J. M. 2014. Bone morphogenetic proteins: facts, challenges, and future perspectives. J. Dent. Res. 93, 335-345.

Celil A.B., Campbell P.G., Datto M., Wang X.F., Hollinger J.O., Selvamurugan N., Kwok S., Alliston T., Reiss M., Partridge N.C., Hassel S., Schmitt S., Hartung A., Roth M., Nohe A., Petersen N., Ehrlich M., Henis Y.I., Sebald W., Knaus P., Lee K.S., Hong S.H., Bae S.C., Lai C.F., Cheng S.L. 2005. BMP-2 and insulin-like growth factor-I mediate Osterix (Osx) expression in human mesenchymal stem cells via the 
MAPK and protein kinase D signaling pathways. $J$. Biol. Chem. 280, 31353-31359.

Chung B.D., Kayserili H., Ai M., Freudenberg J., Uzumcu A., Uyguner O. 2009. A mutation in the signal sequence of LRP5 in a family with an osteoporosispseudoglioma syndrome (OPPG)-like phenotype indicates a novel disease mechanism for trinucleotide repeats. Hum. Mutat. 30, 641-648.

Clarke B. 2008. Normal bone anatomy and physiology. Clin. J. Am. Soc. Nephrol. 3, 131-139.

Crane J. L., \& Cao X. 2014. Function of matrix IGF-1 in coupling bone resorption and formation. J. Mol. Med. 92, 107-115.

Crane, J. L., \& Cao, X. 2014. Bone marrow mesenchymal stem cells and TGF- $\beta$ signaling in bone remodeling. J. Clin. Invest. 124, 466.

Devescovi, V., Leonardi, E., Ciapetti, G., and Cenni, E. 2008. Growth factors in bone repair. Chir. Organi. Mov. 92, 161.

Dinescu S. 2015. Cellular and molecular mechanism of adipose-derived stem cells differentiation. PhD thesis, University of Bucharest, Bucharest, Romania.

Ducy, P. 2000. Cbfa1: a molecular switch in osteoblast biology. Dev. Dyn. 219, 461-471.

Ehlen H.W., Buelens L.A., Vortkamp A. 2006. Hedgehog signaling in skeletal development. Birth Defects Res. C Embryo Today, 78, 267-279.

Engin F. and Lee B. 2010. NOTCHing the bone: Insights into multi-functionality. Bone, 46, 274-280.

Feng X. 2009. Chemical and Biochemical Basis of CellBone Matrix Interaction in Health and Disease. Curr Chem Biol. 3, 189-196.

Gentili C., Cancedda R. 2009. Cartilage and bone extracellular matrix. Curr. Pharm. Des. 15, 1334-1348.

Goulet J.A., Seunas L.E., DeSilva G., Mary Lou V.H. 1997. Autogenous iliac crest bone graft Complications and functional assessment. Clin. Orthop. Relat. Res. 339, 76-81.

Hadijdakis D.J. and Androulakis I.I. 2006. Bone remodeling. Ann N Y Acad Sci. 1092, 385-396.

Hart M.L., Maerz J.K., Aicher W.K. 2012. The International Conference on Tissue Science and Engineering 2012: News on Emerging Cell - Based Therapies?" J. Tissue Sci. Eng. 11, e001.

Hayrapetyan A., Jansen J.A., van den Beucken J. JJP. 2015. Signaling pathways involved in osteogenesis and their application for bone regenerative medicine. Tissue Eng. Part B Rev. 21, 1-42.

Heo J. S., Lee S. G., Kim H. O. 2017. Distal-less

homeobox 5 is a master regulator of the osteogenesis of human mesenchymal stem cells. Int. J. Molec. Med. 40, 1486-1494.

Hill T.P., Spater D., Taketo M.M., Birchmeier W., Hartmann C. 2005. Canonical Wnt/beta-catenin signaling prevents osteoblasts from differentiating into chondrocytes. Dev. Cell, 8, 727-738.

Hilton M.J., Tu X., Wu X., Bai S., Zhao H., Kobayashi T., Kronenberg H.M., Teitelbaum S.L., Ross F.P.,
Kopan. R. 2008. Notch signaling maintains bone marrow mesenchymal progenitors by suppressing osteoblast differentiation. Nat. Med. 306, 14.

Hubbell J.A. 1995. Biomaterials in tissue engineering. Nat. Biotechnol. 13, 565-576.

Hughes F., Turner W., Belibasakis G., Martuscelli G. 2006. Effects of growth factors and cytokines on osteoblast differentiation. Periodontol. 41, 48-72.

Isefuku S., Joyner C. J., Reed A. A. C., Simpson A. H. R. W. 2004. Distraction osteogenesis in the Cbfa-1+/mouse. J. Orthope. Res. 22, 1276-1282.

Jayakumar P., Silvio L.D. 2010. Osteoblasts in bone tissue engineering. Proc. Inst. Mech. Eng. H. 224, 1415-1440.

Jensen E.D., Gopalakrishnan R., Westendorf J.J. 2010. Regulation of gene expression in osteoblasts. Biofactors, 36, 25-32.

Kim J.H., Liu X., Wang J., Chen X., Zhang H., Kim S.H., Cui J., Li R., Zhang W., Kong Y., Zhang J., Shui W., Lamplot J., Rogers M.R., Zhao C., Wang N., Rajan P., Tomal J., Statz J., Wu N., Luu H.H., Hanydon R.C., He T.C. 2013. Wnt signaling in bone formation and its therapeutic potential for bone diseases. Ther. Adv. Muskuloskel. Dis. 5, 13-31.

Kirkham G. R. and Cartmell S. H. 2007. Genes and proteins involved in the regulation of osteogenesis. In: Topics in Tissue Engineering. Ashammakhi N., Reis R. and Chiellini E. (Ed.), Manchester, 1-22.

Kneser U., Schaefer D. J., Munder B., Klemt C., Andree C., Stark G. B. 2002. Tissue engineering of bone. Minim. Invasive Ther. Allied Technol. 11, 107116.

Komori T., 2006. Regulation of osteoblast differentiation by transcription factors. J. Cell Biochem. 99, 12331239.

Komori T., 2010. Regulation of osteoblast and odontoblast differentiation by Runx2.J. Oral Biosci. 52, 22-25.

Komori, T., \& Kishimoto, T. 1998. Cbfa1 in bone development. Curr. Opin. Genet. Dev. 8, 494-499.

Korenberg J.R., Chen X.N., Doege K., Grover J. and Roughley P.J. 1993. Assignment of the human aggrecan gene (AGC1) to $15 \mathrm{q} 26$ using fluorescence in situ hybridization analysis, Genomics 16, 546-548.

Kronenberg H.M. 2003. Developmental regulation of the growth plate. Nature 423, 332-336.

Lamoureux F., Baud'huin M., Duplomb L., Heymann D., Rédini F. 2007. Proteoglycans: key partners in bone cell biology. Bioessays 29, 758 .

Lattanzi W., Bernardini C. 2012. Genes and Molecular Pathways of the Osteogenic Process, Osteogenesis. Biochemistry, Gen. Mol. Biol. 2, 1-37.

Levi, G., Geoffroy, V., Palmisano, G., \& de Vernejoul, M. C. 2002. Bones, genes and fractures. EMBO Rep. 3, 22-26.

Long F., Chung U.I., Ohba S., McMahon J., Kronenberg H.M., McMahon A.P. 2004. Ihh signaling is directly 
required for the osteoblast lineage in the endochondral skeleton. Development 131, 1309-1318.

Luu H.H., Song W.X., Luo X., Manning D., Luo J., Deng Z.L., Sharff K.A., Montag A.G., Haydon R.C., He T.C. 2006. Distinct roles of Bone Morphogenetic Proteins in Osteogenic Differentiation of Mesenchymal Stem Cells. Wiley InterSci. 25, 665-677.

McLarren K.W., Lo R., Grbavec D., Thirunavukkarasu K., Karsenty G., Stifani S. 2000. The mammalian basic helix loop helix protein HES-1 binds to and modulates the transactivating function of the runt-related factor Cbfa1. J. Biol. Chem. 275, 530-538.

Monroe D.G., McGee-Lawrence M.E., Oursler M.J., Westendorf J.J. 2012. Update on Wnt signaling in bone cell biology and bone disease. Gene 492, 1-18.

Nakamura T., Aikawa T., Iwamoto-Enomoto M., Iwamoto M., Higuchi Y., Pacifici M., Kinto N., Yamaguchi A., Noji S., Kurisu K., Matsuya T. 1997. Induction of osteogenic differentiation by hedgehog proteins. Biochem. Biophys. Res. Commun. 237, 465469.

Neen D., Noyes D., Shaw M., Gwilym S., Fairlie N., Birch N., 2006. Healos and bone marrow aspirate used for lumbar spine fusion: a case controlled study comparing healos with autograft. Spine 31, 636-640.

$\mathrm{Ng}$ F., Boucher S., Koh S., Sastry K. S., Chase L., Lakshmipathy U. 2008. PDGF, TGF- $\beta$, and FGF signaling is important for differentiation and growth of mesenchymal stem cells (MSCs): transcriptional profi ling can identify markers and signaling pathways important in diff erentiation of MSCs into adipogenic, chondrogenic, and osteogenic lineages. Blood 112, 295307.

Nga N.K., Hoai T.T., Viet P.H. 2015. Biomimetic scaffolds based on hydroxyapatite nanorod/poly (d, l) lactic acid with their corresponding apatite-forming capability and biocompatibility for bone-tissue engineering. Colloids Surf. B Biointerfaces 128, 506514.

Nguyen L. H., Annabi N., Nikkhah M., Bae H., Binan L., Park S., Khademhosseini, A. 2012. Vascularized bone tissue engineering: approaches for potential improvement. Tissue Eng. Part B Rev. 18, 363-382.

Ornitz, D. M., \& Marie, P. J. 2015. Fibroblast growth factor signaling in skeletal development and disease. Genes Dev. 29, 1463-1486.

Pandolfi L., Minardi S., Taraballi F., Liu X., Ferrari M., Tasciotti E. 2016. Composite microspherefunctionalized scaffold for the controlled release of small molecules in tissue engineering. J. Tissue Eng. 7, 1-11.

Polo-Corrales L., Latorre-Esteves M., Ramirez-Vick J. E. 2014. Scaffold Design for Bone Regeneration. J. Nanosci. Nanotehnol. 14, 15-56.

Quarto N., Behr B., Longaker M.T. 2010. Opposite spectrum of activity of canonical Wnt signaling in the osteogenic context of undifferentiated and differentiated mesenchymal cells: implications for tissue engineering. Tissue Eng. Part A 16, 3185-3197.

Rose F.R., Oreffo R.O. 2002. Bone tissue engineering: hope vs hype. Biochem. Biophys. Res. Commun. 292, 17.

Rutkovskiy A., Stenslokken K.O., Vaage I.J. 2016. Osteoblast Differentiation at a Glance. Med. Sci. Monit. Basic Res. 22, 95-106.

Salgado A. J., Coutinho O. P., Reis R. L. 2004. Bone tissue engineering: state of the art and future trends. Macromol. Biosci. 4, 743-765.

Seeman E. 2009. Bone modeling and remodeling. Crit Rev Eukaryot Gene Expr. 19, 219-233.

Sethi N, Kang Y. 2012. Notch signaling: mediator and therapeutic target of bone metastasis. Bonekey Rep. 3, 1038.

Sinha, K. M., Zhou, X. 2013. Genetic and molecular control of osterix in skeletal formation. J. Cell Biochem. 114, 975-984.

Sodek, J., Ganss, B., \& McKee, M. D. 2000. Osteopontin. Crit. Rev. Oral Biol. Med. 11(3), 279-303.

Tezuka K., Yasuda M., Watanabe N., Morimura N., Kuroda K., Miyatani S., Hozumi N. 2002. Stimulation of osteoblastic cell differentiation by Notch. J. Bone Miner. Res. 17, 231-239.

Van der Horst G., Farih-Sips H., Lowik C.W., Karperien M. 2003. Hedgehog stimulates only osteoblastic differentiation of undifferentiated KS483 cells. Bone 33, 899-910.

Vimalraj, S., Arumugam, B., Miranda, P. J., Selvamurugan, N. 2015. Runx2: Structure, function, and phosphorylation in osteoblast differentiation. Int. J. Biol. Macromol. 78, 202-208.

Vortkamp A., Lee K., Lanske B., Segre G.V., Kronenberg, H.M.,Tabin C.J. 1996. Regulation of rate of cartilage differentiation by Indian Hedgehog and PTH-related protein. Science 273, 613-622.

Williams D. 2004. Benefit and risk in tissue engineering. Mater. Today, 7, 24-29.

Yang J., Philipp A., Ling Y., Ying-Zi Y. 2015. The Hedgehog signaling pathway in bone formation. Int. $J$. Oral Sci. 7, 73-79. 